\title{
Correlation of angiographic scoring system with percutaneous revascularization success in chronic coronary total occlusion patients
}

\begin{abstract}
Background: The percutaneous coronary intervention of chronic total occlusions (CTOs) is considered the most challenging technical procedure in the current interventional cardiology. Japanese Multicenter CTO Registry (J-CTO Score) was established to predict the probability of successful guidewire crossing through a half-hour.
\end{abstract}

Aim: To assess the correlation of $\mathrm{J}$ score with percutaneous revascularization success in chronic coronary total occlusion patients.

Method: This is a retrospective study that was included CTO patients who underwen coronary angiography at Cardiac Catheterization Laboratory at King Abdul-Aziz Cardiac Center between January 2010 - December 2017 will be extracted from an electronic database (Apollo Lx, Best Care, Xcelera, Muse). Data collected from patients included demographics, cardiovascular risk factors, co-morbidities, angiographic parameters, Haemodynamic Measurements, and laboratory tests. SPSS was used to analyze data.

Results: There were 173 patients included in the study, the mean \pm SD of CTOs number was $1.4 \pm 0.8$, the mean $\pm \mathrm{SD}$ of $\mathrm{J}-\mathrm{CTO}$ score was $1.8 \pm 0.9$, the mean $\pm \mathrm{SD}$ of fluro time was $29.3 \pm 14$. There was no significant association between J-CTO score and outcome of patients $(\mathrm{P}=0.6)$, the amount of contrast used also had no significant association with patients'outcome $(\mathrm{P}=0.4)$, whereas the mean of fluro time was significantly associated with outcome of patients $(\mathrm{P}=0.01)$.

Conclusion: J score showed no association with the success rate, however lower fluro time was a predictor of success.

Keywords: J score, success, total occlusion, percutaneous revascularization
Volume I3 Issue 3 - 2020

\author{
Mahammed A Balghaith,' Ibrahim S Alharbi, ${ }^{2}$ \\ Azra Mahmud, ${ }^{3}$ Abdulrhman Alenezi ${ }^{4}$ \\ 'King Abdulaziz Medical City, Saudi Arabia \\ ${ }^{2}$ National Guard Health Affairs, Saudi Arabia \\ ${ }^{3}$ King Abdullah International Medical Research Center, Saudi \\ Arabia \\ ${ }^{4}$ King Saud bin Abdulaziz University for Health Sciences, Saudi
} Arabia

Correspondence: Ibrahim S Alharbi, National Guard Health Affairs, Email Dr.ibrahimal7arbi@gmail.com

Received: June 18,2020 | Published: June 26, 2020

\section{Introduction}

The percutaneous coronary intervention of chronic total occlusions (CTOs) is considered the most challenging technical procedure in the current interventional cardiology. ${ }^{1}$ While there is not enough data collected yet, growing reviews suggest that successful percutaneous CTO revascularization treats symptoms, makes progress in left ventricular systolic function, and decreases the need for surgical coronary bypass. Besides, complete coronary revascularization improves survival. ${ }^{2}$ Yet, compared to individuals with severely stenotic, but patent vessels, individuals with a CTO are more likely to be referred for coronary bypass surgery or medical therapy. In a recently published Canadian registry, only one in ten individuals with a CTO underwent PCI. ${ }^{3}$ It is worth mentioning that the success rate of CTO PCI was about $70 \%$. This doubt related to the likelihood of success, mixed with the fear of high complication rates and beside the procedure costs, contribute to the unwillingness to percutaneously revascularize CTOs. ${ }^{4}$

Japanese Multicenter CTO Registry (J-CTO Score) was initially established to anticipate the probability of successful guidewire crossing within a half-hour. Independent angiographic predictors of failure (each given one point) that pretend the J-CTO score, which comprises angiographic evidence of the prior failed attempt, heavy calcification, blunt proximal stump, bending within the occluded segment, and occlusion length $>=20 \mathrm{~mm} .{ }^{5}$ CTOs were then graded as easy, intermediate, difficult, and very difficult (J-CTO Scores of 0, 1, 2 , and $\geq 3$, respectively). Since then, the J-CTO score has been reported to anticipate the overall probability of CTO PCI success. High J-CTO scores have been reported to associate with lesions complexity, ${ }^{6}$ and may account for the paradox of stagnant CTO PCI success rate over time, which was due to intervening on increasingly complex CTOs. ${ }^{7}$

PCI in chronic total occlusion has expressed significant evolution with the development of new techniques, as well as with the training of specialized personnel. These predictors have significantly raised success rates, making these procedures more efficient and predictable. CTO is known as coronary obstructions, which establish total occlusion of vessel lumen with TIMI 0 flow and duration longer than three months. Occlusions with the minimal passage of contrast without opacification of the distal vessel are considered "functional CTO." $"$

CTOs occur in $18-52 \%$ of individuals submitted to coronary angiography and who have coronary heart disease. ${ }^{9}$ Recent reviews showed a prevalence between 16 and $20 \%{ }^{10}$ In these studies, the percentage of patients with CTO submitted to PCI was low. In two Canadian studies, only $9-10 \%$ of individuals underwent PCI, while 57 to $64 \%$ of them remained in clinical treatment, and 26 to $34 \%$ were referred for surgery. ${ }^{11}$ It is worth mentioning that the J-CTO score, a scale based on pre-procedural angiographic factors, and previous procedural failure, could be used to predict the probability 
of successful guidewire crossing of CTO lesions within 30 min and an improved midterm success rate one year after CTO-PCI. Furthermore, the importance of the J-CTO score to predict the complexity of CTO recanalization was externally validated in a more contemporary cohort. However, the impact of the J-CTO score on the long-term target lesion revascularization (TLR) rate, as well as on other clinical outcomes has yet to be completely evaluated. ${ }^{12}$

Abe et al. ${ }^{12}$ concluded that 425 lesions of 408 participants who subjected successful CTO-PCI over a median follow-up of 63.0 months in the J-CTO Registry. Besides, The cumulative prevalence of TLR of lesions related to a J-CTO score $\geq 2$ was considerably higher than in the others with a J-CTO score $\leq$ one ( 27.0 versus $19.4 \%$ at five years, respectively). Between 323 lesions of 309 participants with a complete five-year follow-up, the rate of TLR was $28 \%$. A J-CTO score $\geq$ two was independently related to a higher risk of target lesion revascularization (odds ratio, 1.73; 95\% confidence interval, 1.01 $2.99, \mathrm{P}=0.048$ ) even after amendment for clinically relevant baseline predictors. $^{12}$

Christopoulos et al..$^{13}$ reported that the effect of the J-CTO score on technical success and procedure time was estimated with univariable logistic and linear regression, respectively. The effectiveness of the logistic regression model was assessed with the Hosmer-Lemeshow statistic and receiver operator characteristic curves. Antegrade wiring techniques were commonly used in easy lesions $(97 \%)$ than very difficult lesions $(58 \%)$, while the retrograde approach became less common with more lesion difficulty $(41 \%$ for very difficult lesions vs. $13 \%$ for easy lesions). The logistic regression model for technical success revealed satisfactory calibration and discrimination ( $\mathrm{p}$ for Hosmer-Lemeshow $=0.743$ and area under curve $=0.705$ ). The J-CTO score was related to a two-fold increase in the odds of a technical failure (odds ratio 2.04, 95\% confidence interval [95\% CI] 1.52-2.80, $\mathrm{p}<0.001)$. Procedure time increased by about 20 minutes for every one-point increase of the J-CTO score (regression coefficient 22.33, $95 \%$ CI 17.45-27.22, $\mathrm{p}<0.001){ }^{13}$

Nombela et al. ${ }^{6}$ concluded that the J-CTO score was applied for each participant, and calibration and discrimination were evaluated in the whole group, and according to the approach (antegrade $47 \%$ and retrograde 53\%). Clinical and angiographic differences were reported between the original and studied group. The median J-CTO score was $2.18 \pm 1.26$, and successful guidewire crossing within a half-hour and final angiographic success was $44.5 \%$ and $90.4 \%$, respectively. The J-CTO score revealed good discrimination (c statistic, $>0.70$ ) and calibration (Hosmer-Lemeshow $\mathrm{P}>0.1$ ) in the whole group and for antegrade and retrograde approaches. However, the final success rate was not related to the J-CTO score. ${ }^{6}$ Morino et al. ${ }^{5}$ concluded that the set endpoint was carried out in $48.2 \%$ of lesions. Independent predictors included bending, calcification, stump, blunt, occlusion length $>20$ $\mathrm{mm}$, and formerly failed lesion. Easy, intermediate, difficult, and very difficult cohorts, stratified by J-CTO score, revealed stepwise, proportioned, and highly reproducible differences in the probability of successful guidewire crossing within half-hour (in the derivation set $87.7 \%, 67.1 \%, 42.4 \%$, and $10.0 \%$ and in the validation set $92.3 \%$, $58.3 \%, 34.8 \%$, and $22.2 \%$, respectively). ${ }^{5}$ This study aims to assess the correlation of $\mathrm{J}$ score with percutaneous revascularization success in chronic coronary total occlusion patients.

\section{Subjects and methods}

Retrospectively study which was conducted on Males and females patients with a diagnosis of CTO who underwent coronary angiography at Cardiac Catheterization Laboratory at King AbdulAziz Cardiac Center. All patients $>18$ years of age, with documented CTO from January 2010 to December 2017 who underwent a successful cardiac catheterization procedure at King Abdul Aziz Cardiac Center were included in the study. We exclude patients whose CTOs in more than one vessel and CTOs in small coronary branch vessels. Random sampling technique.

Retrospectively, all patients who underwent coronary angiography at Cardiac Catheterization Laboratory at King Abdulaziz Cardiac Center between January 2010 - December 2017 were extracted from an electronic database (Apollo Lx, Best Care, Xcelera, Muse).

\section{Data include}

Demographics: age, gender, BMI, BSA, Ethnic group, date of procedure (Apollo Lx, Best Care).

Cardiovascular risk factors: smoking, diabetes, hypertension, dyslipidemia, hyperuricemia, family history of premature cardiovascular disease (Apollo Lx, Best Care).

Co-morbidities: Renal impairment (CKD class), cerebrovascular disease, peripheral vascular disease, significant valvular lesion, heart failure, prior history of revascularization ( $\mathrm{PCi}$ or $\mathrm{CABG}$ ). (Apollo Lx, Best Care).

We classified patients into two groups: those with successful CTOPCI and those with failed CTO-PCI.

Procedural success defined as residual stenosis less than $50 \%$ and wire crossed. After PCI, patients will be followed retrospectively by on regular outpatient visits.

\section{Statistical analysis}

SPSS program will be used for data analysis, version 22.0. Descriptive statistics were done. A P-value of 0.05 or less will be significant.

\section{Results}

We included 173 CTO patients who underwent coronary angiography; the mean $\pm \mathrm{SD}$ of the age of all patients was $60.2 \pm 11.4$ years old. The majority of patients were males $142(82.1 \%)$, and the mean \pm SD of BMI was $30.3 \pm 5.3$. There were $132(76.3 \%), 57(32.9 \%)$, $84(48.6 \%)$ of patients suffering HTN, HF, DM respectively, and there were $43(24.9 \%)$ current smokers. There were $144(83.2 \%)$, $6(3.5 \%), 8(4.6 \%), 6(3.5 \%)$ of patients having DPL, PVD, CVA, Afib, respectively. Prior MI, prior CABAG, prior PCI were found among 103(59.5\%), 22(12.7\%), 51(29.5\%) of patients, respectively. Cardiogenic shock was experienced by $5(2.9 \%)$ of patients, whereas CKD was experienced by $22(12.7 \%)$, whereas $7(4 \%)$ of patients were hemodialysis patients. The amount of contrast used ranged from 20500 with a median of 145 and a mean \pm SD of $162.7 \pm 82.9$. The fluro time ranged from 2.9-85 with a median of 26.4 and the mean $\pm \mathrm{SD}$ of $29.3 \pm 14$. The wire was crossed in $126(72.8 \%)$ cases. The details of the demographics and clinical characteristics of patients are shown in Table1 \& 2.

The angiographic factors affecting the success rate are shown in table 3; the success and failure outcome didn't differ with J-CTO score $(\mathrm{P}=0.65)$. Also, amount of contrast used had no significant effect on the success and failure rate $(\mathrm{P}=0.42)$, whereas fluro time significantly affected the success rate $(\mathrm{P}=0.01)$, where the mean of fluro time was lower in patients experienced successful procedure (Figure 1). 
Table I Demographics and clinical characteristics of patients

\begin{tabular}{|c|c|}
\hline Variables & $\operatorname{Description}(n=\mid 73)$ \\
\hline \multicolumn{2}{|l|}{ Age } \\
\hline Range & $36-85$ \\
\hline Mean \pm SD & $60.2 \pm 11.4$ \\
\hline Median(IQR) & $6 I(52-69)$ \\
\hline \multicolumn{2}{|l|}{ Sex } \\
\hline Male & $142(82.1)$ \\
\hline Female & $3 I(17.9)$ \\
\hline \multicolumn{2}{|l|}{ BMI } \\
\hline Range & $15.8-49.2$ \\
\hline Mean \pm SD & $30.3 \pm 5.3$ \\
\hline Median(IQR) & $30.4(26.6-33.6)$ \\
\hline HTN & $132(76.3)$ \\
\hline $\mathrm{HF}$ & $57(32.9)$ \\
\hline DM & $84(48.6)$ \\
\hline \multicolumn{2}{|l|}{ Smoker } \\
\hline Non-smokers & I23(7I.I) \\
\hline Current smoker & $43(24.9)$ \\
\hline Ex-smoker & $7(4)$ \\
\hline DLP & I44(83.2) \\
\hline PVD & $6(3.5)$ \\
\hline CVA & $8(4.6)$ \\
\hline Valve surgery & $0(0)$ \\
\hline Afib & $6(3.5)$ \\
\hline Prior MI & $103(59.5)$ \\
\hline Prior CABG & $22(12.7)$ \\
\hline Prior PCl & $5 \mathrm{I}(29.5)$ \\
\hline Cardiogenic shock & $5(2.9)$ \\
\hline CKD & $22(12.7)$ \\
\hline Hemodialysis & $7(4)$ \\
\hline \multicolumn{2}{|l|}{ Dx } \\
\hline SA & $72(4 I .6)$ \\
\hline NSTEMI & $45(26)$ \\
\hline UA & $35(20.2)$ \\
\hline STEMI & $16(9.2)$ \\
\hline $\mathrm{SU} / \mathrm{HF}$ & $3(1.7)$ \\
\hline Elective AICD & $2(1.2)$ \\
\hline
\end{tabular}

Table 2 Regarding the success rate was $50.3 \%$, whereas the failure rate was $49.7 \%$, Figure I

\section{J- CTO score}

$\begin{array}{ll}\text { Range } & 0-4 \\ \text { Mean } \pm \text { SD } & 1.8 \pm 0.9 \\ \text { Median(IQR) } & 2(I-2) \\ \text { Amount of contrast used } & \\ \text { Range } & 20-500 \\ \text { Mean } \pm S D & 162.7 \pm 82.9 \\ \text { Median(IQR) } & 145(110-200) \\ \text { Fluro time } & \\ \text { Range } & 2.9-85 \\ \text { Mean } \pm S D & 29.3 \pm 14 \\ \text { Median(IQR) } & 26.4(20-37)\end{array}$

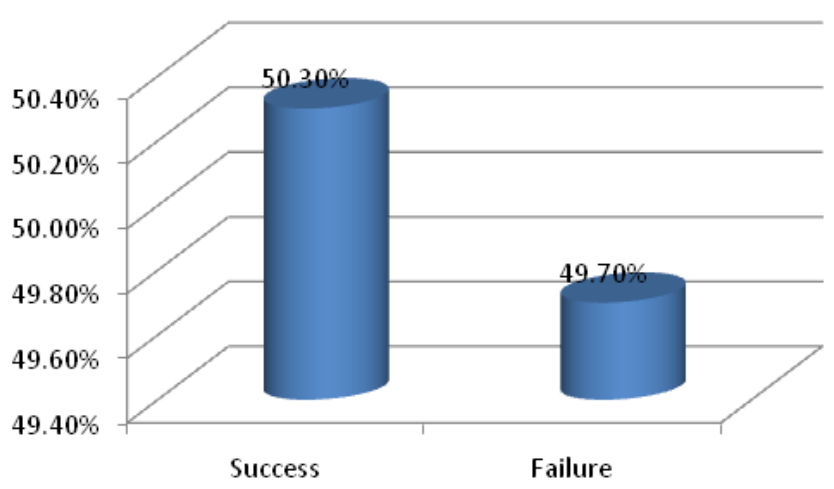

Figure I Outcome of patients.

\section{Discussion}

Planning of the recanalization procedure requires the use of angiographic scores to estimate the probable success rate and the type of approach. ${ }^{8}$ The J-CTO score is the most widespread and oldest one.15 Higher J-CTO score significantly indicates lower success rates, greater use of contrast, more frequent use of retrograde approach and longer procedures. ${ }^{13,14}$ In the present study, we aimed to investigate the association of $\mathrm{J}$ score with percutaneous revascularization success in chronic coronary total occlusion patients. The range of J-CTO score was $0-4$ with a mean of 1.8 , the mean amount contrasted used, and fluro time was 162.7 and 29.3 , respectively. The wire was crossed among $72.8 \%$ of patients; the success rate of the procedure was $50.3 \%$. By investigating the factors affecting the outcome of patients, it was found that J-CTO score had no association with the outcome of patients the mean of J-CTO score was similar in success group and failure group (1.8); also the mean of amount of contrast used didn't differ between the two groups. However, fluro time showed a significant influence on the outcome of patients; the low mean of fluro time was significantly associated with the success of the procedure (Table 3). 
Table 3 Factors affecting success

\begin{tabular}{|c|c|c|c|}
\hline & Outcome & & \\
\hline Factors & Success $(n=87)$ & $\begin{array}{l}\text { Failure } \\
(n=86)\end{array}$ & $P$ value \\
\hline \multicolumn{4}{|l|}{ J- CTO score } \\
\hline Range & $0-4$ & $0-4$ & \\
\hline Mean \pm SD & $1.8 \pm 0.8$ & $1.8 \pm 1$ & \\
\hline Median(IQR) & $2(I-2)$ & $2(I-2)$ & 0.659 \\
\hline \multicolumn{4}{|c|}{$\begin{array}{l}\text { Amount of } \\
\text { contrast used }\end{array}$} \\
\hline Range & $30-500$ & $20-500$ & \\
\hline Mean $\pm S D$ & $165.7 \pm 80$ & $159.6 \pm 86.2$ & \\
\hline Median(IQR) & $150(110-210)$ & $140(\mid 10-190)$ & 0.424 \\
\hline \multicolumn{4}{|l|}{ Fluro time } \\
\hline Range & $7-80$ & $2.9-85$ & \\
\hline Mean \pm SD & $26.8 \pm 13$ & $31.9 \pm 14.7$ & \\
\hline Median(IQR) & $24(19-35.1)$ & $31.4(23-38)$ & 0.010 \\
\hline
\end{tabular}

It was demonstrated that $\mathrm{J}$-CTO score $\geq 3$ was independently associated with worse outcomes in the long term after retrograde CTO PCI. ${ }^{15}$ In one study, ${ }^{16}$ it was found that $36.3 \%$ of patients were presented with $\mathrm{J}-\mathrm{CTO}$ score 2 , and the higher score was associated with higher failure rates $(\mathrm{P}=0.006)$. Another study $^{17}$ reported that the success rate of CTO-PCI was high $82.6 \%$, which was higher than our rate. However, a fewer rate of $29.4 \%$ of cases achieved 30 min wire crossing, whereas, in our study, the wire crossing was a success in $72.8 \%$. The previous study ${ }^{17}$ revealed that the CTA-derived J-CTO score was more useful than the J-CTO score derived from Conventional angiography in predicting the success of the procedure and 30minutes crossing.

It was reported that high J-CTO score lesions had a higher fiveyears risk of TLR, ${ }^{12}$ and the J-CTO score was strongly associated with final success rate and efficiency, and J-CTO was supported to be used in CTO interventions. ${ }^{13}$ It was shown that the J-CTO score helped to predict the complexity of CTO recanalization. ${ }^{6}$

\section{Conclusion}

In the present study, we found that the success rate was low and J-score, as well as the amount of contrast couldn't predict the success rate and their mean value didn't vary between the patients in success group and failed group, whereas low fluro time can be a predictor of the success of the process.

\section{Acknowledgments}

None.

\section{Conflicts of interest}

The authors declare there are no conflicts of interest related to the article.

\section{Funding}

None.

\section{References}

1. Grantham JA, Marso SP, Spertus J, et al. Chronic total occlusion angioplasty in the United States. JACC: Cardiovascular Interventions. 2009;2(6):479-486.

2. Joyal D, Afilalo J, Rinfret S. Effectiveness of recanalization of chronic total occlusions: a systematic review and meta-analysis. Am Heart J. 2010;160(1):179-187.

3. Fefer P, Knudtson ML, Cheema AN, et al. Current perspectives on coronary chronic total occlusions: the Canadian Multicenter Chronic Total Occlusions Registry. J Am Coll Cardiol. 2012;59(11):991-997.

4. PrasadA, Rihal CS, Lennon RJ, et al. Trends in outcomes after percutaneous coronary intervention for chronic total occlusions: a 25-year experience from the Mayo Clinic. J Am Coll Cardiol. 2007;49(15):1611-1618.

5. Morino $\mathrm{Y}$, Abe M, Morimoto $\mathrm{T}$, et al. Predicting successful guidewire crossing through chronic total occlusion of native coronary lesions within 30minutes: the J-CTO (Multicenter CTO Registry in Japan) score as a difficulty grading and time assessment tool. JACC: Cardiovascular Interventions. 2011;4(2):213-221

6. Nombela-Franco L, Urena M, Jerez-Valero M, et al. Validation of the J-chronic total occlusion score for chronic total occlusion percutaneous coronary intervention in an independent contemporary cohort. Circulation: Cardiovascular Interventions. 2014;6(6):635-643.

7. Syrseloudis D, Secco GG, Barrero EA, et al. Increase in J-CTO lesion complexity score explains the disparity between recanalisation success and evolution of chronic total occlusion strategies: insights from a singlecentre 10-year experience. Heart. 2013;99(7):474-479.7

8. Ybarra LF, Cantarelli MJ, Lemke VM, et al. Percutaneous Coronary Intervention in Chronic Total Occlusion. Arq Bras Cardiol. 2018;110(5):476-483.

9. Christofferson RD, Lehmann KG, Martin GV, et al. Effect of chronic total coronary occlusion on treatment strategy. Am J Cardiol. 2005;95(9):10881091 .

10. Fefer, P., Knudtson, M. L., Cheema, A. N., Galbraith, P. D., Osherov A. B., Yalonetsky, S., ... \& Sparkes, J. D. (2012). Current perspectives on coronary chronic total occlusions: the Canadian Multicenter Chronic Total Occlusions Registry. Journal of the American College of Cardiology, 59(11):991-997?

11. Azzalini L, Jolicoeur EM, Pighi M, et al. Epidemiology, management strategies, and outcomes of patients with chronic total coronary occlusion. Am J Cardiol. 2016;118(8):1128-1135.

12. Abe M, Morimoto T, Morino Y, et al. Association between J-CTO score and long-term target lesion revascularization rate after successful chronic total coronary occlusion angioplasty (from the J-CTO Registry). Catheter Cardiovasc Interv. 2019;93(6):1025-1032.

13. Christopoulos G, Wyman RM, Alaswad K, et al. Clinical Utility of the J-CTO Score in Coronary Chronic Total Occlusion Interventions: Results from a Multicenter Registry. Circ Cardiovasc Interv. 2015;8(7):e002171.

14. Karacsonyi J, Karatasakis A, Karmpaliotis D, et al. Effect of previous failure on subsequent procedural outcomes of chronic total occlusion percutaneous coronary intervention (from a Contemporary Multicenter Registry). Am J Cardiol. 2016;117(8):1267-1271.

15. Galassi AR, Sianos G, Werner GS, et al. Retrograde recanalization of chronic total occlusions in Europe: procedural, in-hospital, and longterm outcomes from the multicenter ERCTO registry. J Am Coll Cardiol. $2015 ; 65(22): 2388-2400$. 
16. Mehta AB, Mehta N, Chhabria R, et al. Predictors of success in percutaneous Coronary intervention for chronic total occlusion. Indian Heart J. 2018;70((Suppl 3):S269-S274.
17. Fujino A, Otsuji S, Hasegawa K, et al. Accuracy of J-CTO score derived from computed tomography versus angiography to predict successful percutaneous coronary intervention. JACC: Cardiovascular Imaging. 2018;11(2 Part 1):209-217. 